\title{
Stock Management in Hospital Pharmacy using Chance-Constrained Model Predictive Control ${ }^{\text {th }}$
}

\author{
I. Jurado ${ }^{a}$, J. M. Maestre ${ }^{b 1}$, P. Velarde ${ }^{b}$, C. Ocampo-Martinez ${ }^{c}$, I. Fernández ${ }^{d}$, B. Isla \\ Tejera $^{d}$, J. R. del Prado ${ }^{e}$ \\ ${ }^{a}$ Departamento de Matemáticas e Ingeniería, Universidad Loyola Andalucía, Campus Palmas Altas, C/ \\ Energía Solar 1, Edificio G, 41014 Sevilla, Spain. \\ ${ }^{b}$ Departamento de Ingeniería de Sistemas y Automática Universidad de Sevilla, 41092 Sevilla, Spain. \\ ${ }^{c}$ Automatic Control Department, Universitat Politècnica de Catalunya, Institut de Robòtica i Informàtica \\ Industrial (CSIC-UPC), Llorens i Artigas, 4-6, 08028 Barcelona, Spain. \\ ${ }^{d}$ Pharmacy Department, Hospital San Juan de Dios, Córdoba, Spain. \\ ${ }^{e}$ Pharmacy Department, Hospital Universitario Reina Sofía, Córdoba, Spain.
}

\begin{abstract}
One of the most important problems in the pharmacy department of a hospital is stock management. The clinical needs of drugs must be satisfied with limited work labor while minimizing the use of economical resources. The complexity of the problem resides in the random nature of the drug demand and the multiple constraints that must be taken into account in every decision. In this article, chance-constrained model predictive control is proposed to deal with this problem. The flexibility of model predictive control allows taking into account explicitly the different objectives and constraints involved in the problem while the use of chance constraints provides a trade-off between conservativeness and efficiency. The solution proposed is assessed to study its implementation in two Spanish hospitals.
\end{abstract}

Keywords: Hospital Pharmacy, Inventory management, Model Predictive Control, chance constraints

\section{Introduction}

Stock management is a common problem that is present in almost all the companies and organizations. The solution for this problem is given by a policy that determines

\footnotetext{
The authors would like to acknowledge Junta de Andalucía (Pharmacontrol Project, P12-TIC-2400), for funding this work.

Email addresses: i jurado@uloyola.es (I. Jurado ${ }^{a}$ ), pepemaestre@us.es (J. M. Maestre ${ }^{b}$ ), pvelarde@us.es (P. Velarde ${ }^{b}$ ), cocampoeiri.upc.edu (C. Ocampo-Martinez ${ }^{c}$ ),

MariaIsabel.Fernandez@sjd.es (I. Fernández ${ }^{d}$ ), beatrizislatj@gmail.com (B. Isla

Tejera $^{d}$ ), joser.prado.sspa@ juntadeandalucia.es (J. R. del Prado ${ }^{e}$ )

${ }^{1}$ Corresponding author
} 
how and when the orders should be placed. However, there are different difficulties associated to the problem. In the first place, there are uncertainties in the demand and delays in the deliveries, which make the problem not deterministic and require a degree of conservatism to avoid stockouts. It is needless to say that the lack of certain drugs in a hospital may endanger the life of the inpatients and, in the worst case, may have catastrophic consequences in the form of human losses. In order to avoid this situation, it is preferred to increase stock levels, but this is not always possible due to economical constraints. Actually, the pharmacy is a major source of expenses in hospitals. In [1], it is estimated that about $20-35 \%$ of the goods budget of a public hospital is spent by the pharmacy department. In a wider sense, the limitations imposed by the budget are also translated into the human resources in the pharmacy and the room available for storing drugs, which introduce additional constraints for the management. Hence, it may not be possible to place and receive orders too often due to the lack of pharmacists. Likewise, space constraints are important for example in drugs that must be stored in a fridge. Therefore, there is a need to develop advanced cost-efficient safe policies for stock management in hospitals capable of dealing with many different types of constraints.

In general, simple methods are used to solve inventory control problems. A usual policy is that of reorder point $(s, S)$, that is, whenever the stock is below the level $s$, an order is placed to increase the stock up to the value $S$. Another option is to fix a size for the orders, $Q$, and submit an order once the stock is at level $s$. Other related policies about how to solve this problem are given in [2] and [3]. The major drawback with these techniques is that they are not able to take into account all the factors involved in the decision problem. In the literature, other alternatives are also proposed. For example, [4] presents an analytical model for the coordination of inventory and transportation in supply-chain systems. In [5], a supply chain network model consisting of manufacturers and retailers, where the demand is random, is developed. More strategies are presented in [6], where a competitive and cooperative selection of inventory policies in a supply chain with stochastic demand are studied. On the other hand, [7] develops a model to design a supply chain network with deterministic demand.

In this work, a model predictive control (MPC) strategy is proposed given the flexibility offered by its framework to handle multi-variable interactions, constraints on the problem variables, and optimization requirements in a systematic manner. Moreover, MPC has been successfully applied in the industry [8] and in similar problems. Some works based on the application of MPC are, for example, [9] and [10], where MPC is used to supply chain management in semiconductor manufacturing. Another example can be found in [11], where a distributed MPC algorithm with low communication burden is tested by using the MIT Beer Game (a supply chain benchmark). An extension of this scheme for systems with more than two controllers is also tested with supply chains in [12]. In [13], a robust MPC technique is used in a production-inventory system. Finally, in [14] the problem of managing inventories and supply chains is treated to reduce the number of tuning parameters with a technique based on a variation of MPC.

In the particular case of the stochastic control problems, i.e., those where the system being controlled is subject to uncertainties and/or unknown disturbances, the control policy can guarantee that the actual variables do not violate the constraints at the cost of an additional conservatism. That is, the actions implemented by the control policy are 
designed to deal with worst-case scenarios, which results in a waste of resources [15]. In this situation, it is acceptable to assume a low level of risk to save resources. To this end, the original constraints of the problem can be formulated in a probabilistic manner. The use of chance constraints was introduced in [16] and has been studied in a stochastic programming framework [17].

The implementation of MPC in combination with chance constraints is known as chance-constrained MPC (CC-MPC). The rationale of this approach is to replace hard constraints with probabilistic constraints and the nominal cost function with its expected value in the MPC formulation [18], leading to a stochastic optimization problem. CC-MPC offers advantages as robustness, flexibility, low computational requirements, and the possibility of including the level of reliability associated with the constraints [19, 20]. Furthermore, since CC-MPC takes into account the expected performance of the closed loop with probabilistic constraints instead of directly trying to assure robust constraint satisfaction, it avoids the conservatism present in other robust MPC techniques, e.g.: [21, 22].

There are other alternatives in the stochastic MPC literature that are also suitable for this type of problem. One option to deal with constraints on the inputs and states while optimizing some performance criterion, also in the presence of uncertainties or disturbances, is the scenario-based MPC method proposed in [23]. This method is based on the optimization of the control inputs over a finite horizon, subject to robust constraints under a finite number of random scenarios of the uncertainty and/or disturbances. A different but related approach is tree-based MPC [24], where the disturbances are grouped into a rooted tree that branches as the uncertainty grows. A tree of control actions is calculated to match the disturbance tree by the MPC controller. A simpler method is multiple MPC, which is given in [25], where control actions are calculated weighting the probability of occurrence of three possible scenarios. While all these approaches could be valid in for the problem considered in this article, they present some disadvantages with respect to CC-MPC. In the first place, scenario-based MPC requires a great amount of historical data to provide a low risk level. Tree-based MPC also requires a amount of historical data and solves a problem with a larger number of optimization variables, i.e., the computational burden of this method is greater. Finally, multiple MPC oversimplifies the computation of the control actions due to the low number of scenarios considered. In addition, all these methods have in common that the existence of a very severe scenario may result in an increase of conservativeness [26].

In this paper, which is an extension of the previous works [27, 28], CC-MPC is used to solve the problem of inventory management in hospital pharmacies. In particular, the formulation of the problem is generalized with respect to the aforementioned works, where a Gaussian behavior of the demand is assumed. The mathematical development of the controller presented here can be applied even if the demand is only characterized statistically based on historical data. Likewise, the case of several hospitals that cooperate in order to relax their risk levels is another contribution of this work. It must be also remarked that this article has been carried out in the context of a project named Pharmacontrol, whose goal is to improve the inventory management in two Spanish hospitals.

The remainder of the paper is organized as follows. First, a description of the phar- 


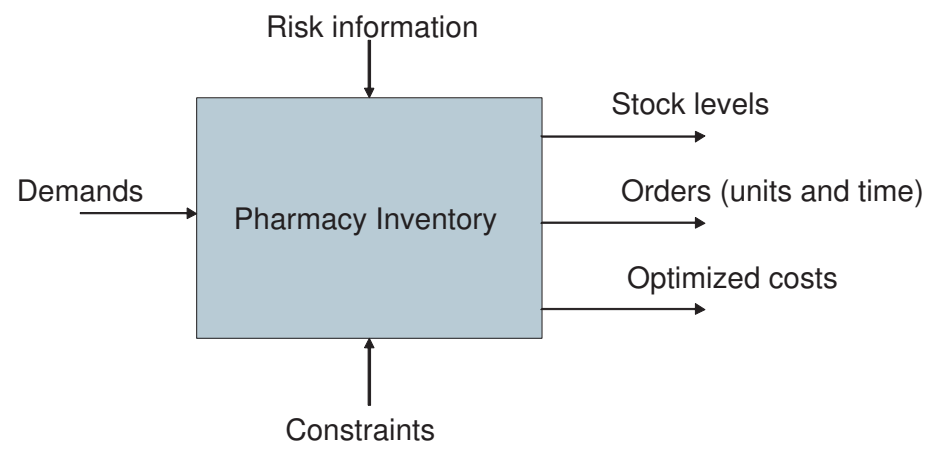

Figure 1: Pharmacy inventory as a system.

macy inventory management optimization problem is shown in Section 2 Section 3 presents the MPC statement for this problem. In Section 4, some simulations are shown and the corresponding results are discussed. Finally, in Section 5 the conclusions are drawn.

\section{Pharmacy Inventory Management}

In this section, the mathematical background needed to build the optimization problem to be solved by the CC-MPC is presented.

\subsection{System Definition}

In general, it will be assumed that there are $N_{i}$ different drugs in the pharmacy inventory. The stock level of each one follows an evolution depending on the orders and on the demand. This evolution is represented by a discrete linear model, which for the particular case of drug $i$ is

$$
s_{i}(t+1)=s_{i}(t)+\sum_{j=1}^{n p_{i}} o_{i}^{j}\left(t-\tau_{i}^{j}\right)-d_{i}(t),
$$

where $s_{i} \in \mathbb{Z}$ is the stock of drug $i, o_{i}^{j} \in \mathbb{Z}$ is the number of ordered items to the $j$-th of the $n p_{i}$ providers of the drug $i, \tau_{i}^{j}$ is its corresponding transport delay, and $d_{i}(t)$ represents the aggregate demand of drug $i$.

The number of ordered items can be modeled as $o_{i}^{j}=\delta_{i}^{j}\left(t-\tau_{i}^{j}\right) o_{i}^{j}\left(t-\tau_{i}^{j}\right)$, where $\delta_{i}^{j}(t)$ is a Boolean variable, that is, $\delta_{i}^{j}(t)=1$ if an order of drug $i$ to provider $j$ is placed during time $t$, otherwise $\delta_{i}^{j}(t)=0$, and $o_{i}^{j} \in \mathbb{Z}$ represents the number of ordered items of drug $i$ to provider $j$, only in those cases where $\delta_{i}^{j}(t)=1$. 


\subsection{Single Hospital Optimization Problem}

The system can be represented according to Figure 1. In this figure, the inputs represent the elements considered by the pharmacy managers to make the decisions about the order placement: the estimated demand, information about potential risks, and the constraints. The outputs are the optimal stock levels, minimum costs, and data about when and how many orders should be placed.

Every time an order is placed, the following costs are involved:

- $p_{i}^{j}$, which is price offered by the $j$-th provider for drug $i$. It is supposed in this paper that it does not depend on the number of items ordered.

- $C_{s h, i}^{j}$, which is the drug $i$ shipping cost when requested from provider $j$.

- $C_{o p, i}$, which is the cost of ordering drug $i$.

- $C_{o s, i}$ is a cost that penalizes when the stock of drug $i$ is below the minimum safety level allowed. This situation is particularly dangerous since there is a high risk for the hospital of running out of drug $i$. In case of need, it is possible to ask another hospital for a loan.

- $C_{s, i}$ is the cost of storage of drug $i$.

- $C_{o, i}$ is the opportunity cost of having drug $i$ in the pharmacy storage.

Note that both stock levels and costs are a direct consequence of the orders placed. Finally, the goals of the managers are the following: i) the demand has to be satisfied; ii) the fixed assets must be reduced; and iii) the number of orders placed has to be minimized. These goals are considered in the optimization problem. In particular, the performance index considered in this work involves a multicriteria weighted function where demand satisfaction, expenses and number of orders are included, i.e.,

$$
\min _{o} J:=\beta_{1} J_{1}(o, t)+\beta_{2} J_{2}(o, t)+\beta_{3} J_{3}(o, t),
$$

where $J_{1}, J_{2}$ and $J_{3}$ are the terms associated to demand satisfaction, costs, and orders, respectively. The weights $\beta_{1}, \beta_{2}$, and $\beta_{3}$ prioritize the different terms and have a strong influence in the solution of the problem.

The terms in the objective function (2) are described next in decreasing priority:

1. $J_{1}$ : Demand satisfaction. The main objective is the minimization of stockout probability. The main issue here is that the demand is not known in advance, i.e., it is stochastic. There may also be uncertainty in the transport delays. For this reason, it is usual in practice to set a safety stock to mitigate the impact of uncertainty. There are two possibilities in the way the safety stock is set: it can be either fixed or variable. The former proposes that the minimum stock level is introduced as a fixed parameter in the optimization problem. The latter treats the safety stock as an optimization variable. This term is expressed as

$$
\min _{\delta_{i}^{j}, o_{i}^{j} \forall i, j} \sum_{k=0}^{N} \sum_{i=1}^{N_{i}} C_{o s, i} \lambda_{\text {stockout }}^{i},
$$


with

$$
\lambda_{\text {stockout }}^{i}= \begin{cases}1 & \text { if } s_{i}(t+k)<\mathrm{S}_{\min }^{i}, \\ 0 & \text { if } s_{i}(t+k)>\mathrm{S}_{\min }^{i},\end{cases}
$$

where $\lambda_{\text {stockout }}^{i}$ indicates whether the safety stock condition is violated, $\mathrm{S}_{\min }^{i}$ is the minimum stock level allowed for the drug $i, N$ is the length of the time horizon in which the condition has to be satisfied, and $N_{i}$ is the number of different drugs.

2. $J_{2}$ : Expenses. This term deals with the minimization of the expenses in the orders of drugs and the inventory levels, i.e.,

$$
\begin{aligned}
\min _{\delta_{i}^{j}, o_{i}^{j}} & \sum_{\forall i, j}^{N} \sum_{k=0}^{N_{i}} \sum_{i=1}^{n p_{i}} \delta_{j=1}^{j}(t+k)\left(p_{i}^{j} o_{i}^{j}(t+k)+C_{s h, i}^{j}\right) \\
& +\sum_{k=0}^{N} \sum_{i=1}^{N_{i}} C_{s, i} s_{i}(t+k)+\sum_{k=0}^{N} \sum_{i=1}^{N_{i}} C_{o, i} s_{i}(t+k) .
\end{aligned}
$$

3. $J_{3}$ : Orders. This term seeks the minimization of the number of placed. It is necessary because placing an order involves a certain cost. Likewise, the work burden for the staff in the pharmacy department is related to this term. For example, in a hospital such as Reina Sofía (Córdoba, Spain) more than twelve thousand orders are placed during a year. Mathematically, it is formulated as:

$$
\min _{\delta} \sum_{k=0}^{N} \sum_{i=1}^{N_{i}} \sum_{j=1}^{n p_{i}} C_{o p, i} \delta_{i}^{j}(t+k) .
$$

Furthermore, the following constraints are considered:

- Storage constraints. As explained before, the level of the stock of drug $i$ has to be greater than a safety stock $S_{\min }^{i}$ to minimize the risk of stockout. In addition, the space restrictions in the storage room must also be considered, which limits the maximum number of drugs that can be stored in the pharmacy. Therefore,

$$
s_{i} \in\left[\mathrm{S}_{\min }^{i}, \mathrm{~S}_{\max }^{i}\right] .
$$

- Order constraints. In order to formulate these constraints, two type of variables are going to be used. The first one is a Boolean variable $\delta_{i}^{j}(t) \in[0,1]$, where the value 1 means that an order of drug $i$ has been placed to provider $j$ during time $t$, and the value 0 means that no order has been placed. In case of placing an order $\left(\delta_{i}^{j}(t)=1\right)$, another variable that represents the ordered number of items is used. This variable should be bounded by both a minimum and a maximum values, i.e.,

$$
o_{i}^{j} \in\left[\min _{o_{i}^{j}}, \max _{o_{i}^{j}}\right] .
$$

There are also some considerations about the minimum number of items to order: 
- The distributors do not work if the number of items ordered is too low. Hence, there is a minimum of items to order at each time, $\min _{\text {item }_{i}^{j}}$. Likewise, the pharmaceutical laboratories require a minimum amount of money to be spent. That is translated into a minimum order size, $\min _{1 a b_{i}^{j}}$. Taking into account these quantities,

$$
\min _{o_{i}^{j}}=\max \left(\min _{\operatorname{item}_{i}^{j}}, \min _{\operatorname{lab}_{i}^{j}}\right) .
$$

- Non-working days of the laboratory (e.g., Sundays, holidays) must be taken into account, which leads to the following constraint:

$$
\delta_{i}^{j}(t)=0, \quad \forall t \notin\{\text { working days }\} .
$$

- Operational constraints. These constraints take into account the limited capacity of the pharmacy for placing orders and receiving shipments. This fact limits the number of orders placed along a time horizon of length $N$, i.e.,

$$
\sum_{k=0}^{N} \sum_{j=1}^{n p_{i}} \delta_{i}^{j}(t+k) \leq \Delta_{i},
$$

where $\Delta_{i}$ is the maximum number of orders of drug $i$ that can be placed along $N$.

- Economical constraints. The money spent during the time horizon $N$ is also limited, being $\max _{\$}$ the maximum amount. The constraint is expressed as

$$
\sum_{k=0}^{N} \sum_{i=1}^{N_{i}} \sum_{j=1}^{n p_{i}} \delta_{i}^{j}(t+k)\left(p_{i}^{j} o_{i}^{j}(t+k)+C_{s h, i}^{j}+C_{o p, i}\right) \leq \max _{\S} .
$$

\subsection{Cooperation between different hospitals}

Different hospitals can cooperate to reduce their safety stocks in case they are close and the consumption of certain drugs is uncorrelated between them. Consequently, the expenses derived from loans between them are low or can even be neglected. This way, the hospitals can focus on the joint stockout probability instead of the individual one, which should be higher, resulting in lower safety and average stock levels. In the simplest case, each hospital would solve its original optimization problem with different constraints. Likewise, it is also possible to formulate this problem in the context of distributed control, where the hospitals are agents that have to reach a consensus on the safety levels. The optimization problem is

$$
\min _{o} \sum_{h=1}^{H} J_{h},
$$


where $J_{h}$ stands for the cost of each hospital and $H$ is the number of collaborating hospitals. The overall objective function taking is given by

$$
J_{h}=\sum_{i=1}^{3} \sum_{j=1}^{H} \beta_{i, j} J_{i, j}(o, t)
$$

where the demand, expenses, and orders terms are like in (3)-(6). The difference here is that, in the demand term, the probability $\operatorname{Pr}\left(s_{i}^{h}(t+k)<0\right)$ can be greater.

The constraints are also the same, (7)-(11), and:

$$
s_{i}^{h}(t+1)=s_{i}^{h}(t)+\sum_{j=1}^{n p_{i}} o_{i}^{j, h}\left(t-\tau_{i}^{j}\right)-d_{i}^{h}(t), \quad \forall h \in\{1, \ldots, H\} .
$$

\section{Model Predictive Control}

The control strategy used to solve the stock management problem is MPC. This strategy is a control method based on output predictions over a prediction horizon $(N)$ calculated using a model of the process [8]. From the optimization of the objective function subject to constraints, a set of future control signals is obtained. Only the control value computed for the time instant $k$ is applied to the process, the rest of them are discarded. This process is repeated in a receding horizon fashion.

\subsection{MPC Setup}

In this section, the implementation of the control problem will be detailed. The objective is the minimization of the objective function (2). Consider the system defined by

$$
s(t+1)=s(t)+o(t-\tau)-d(t)
$$

where $s(t)=\left[s_{1}(t), \ldots, s_{N_{i}}(t)\right], d(t)=\left[d_{1}(t), \ldots, d_{N_{i}}(t)\right]$ and $o(t-\tau)=\sum_{j=1}^{n p_{i}} \delta_{i}^{j}(t-$ $\left.\tau_{i}^{j}\right) u_{i}^{j}\left(t-\tau_{i}^{j}\right)$ represents the total number of items ordered. Note that system 14 is equivalent to (1).

The control variables taken into account in this problem are $\delta_{i}^{j}(t)$ and $o_{i}^{j}(t)$, both components of the control variable $o(t)$. Solving the optimization problem by using directly the control variable $o(t)$ (i.e., $\delta_{i}^{j}(t)$ and $o_{i}^{j}(t)$ together) is a difficult task, since they have different nature because $\delta_{i}^{j}(t)$ is a Boolean variable. The way to proceed will be the use of an exhaustive search algorithm, which will solve the problem as many times as possible scenarios depending on the value of $\left\{\delta_{i}^{j}(t), \ldots, \delta_{i}^{j}(t+N)\right\}$, i.e., $2^{n p_{i} \times N_{i}(N+1)}$ times. In that way, the optimization problem can be solved with respect to the variable $o_{i}^{j}(t)$.

It is straightforward to see that if $\delta_{i}^{j}(t+k)=0$, for $k \in\{0,1, \ldots, N\}$, the quantity of ordered items is $o_{i}^{j}(t+k)=0$. Furthermore, the variable $o_{i}^{j}(t)$ can be considered as a real one to relax the problem. Notice that the solution obtained is still integer due to 
the problem features. In addition, the vector of control variables $\left\{o_{i}^{j}(t), \ldots, o_{i}^{j}(t+N)\right\}$ is reduced by eliminating the components $o_{i}^{j}(t+k)$ that are equal to zero. Hence,

$$
\forall \delta_{i}^{j}(t+k)=0, \quad k \in\{0,1, \ldots, N\}
$$

then

$$
\underbrace{\left[\begin{array}{c}
o_{i}^{j}(t) \\
\vdots \\
o_{i}^{j}(t+k) \\
\vdots \\
o_{i}^{j}(t+N)
\end{array}\right]}_{\mathbf{o}_{\mathbf{i}}^{\mathbf{j}}(\mathbf{t})} \rightarrow \underbrace{\left[\begin{array}{c}
o_{i}^{j}(t) \\
\vdots \\
o_{i}^{j}(t+k-1) \\
o_{i}^{j}(t+k+1) \\
\vdots \\
o_{i}^{j}(t+\tilde{N})
\end{array}\right]}_{\tilde{o}_{\mathbf{i}}^{\mathbf{j}}(\mathbf{t})}
$$

where $\mathbf{o}_{\mathbf{i}}^{\mathbf{j}}(\mathbf{t}) \in \mathbb{R}^{\mathbf{N}+\mathbf{1}}$ and $\tilde{\mathbf{o}}_{\mathbf{i}}^{\mathbf{j}}(\mathbf{t}) \in \mathbb{R}^{\tilde{\mathbf{N}}+\mathbf{1}}$ is a reduced vector of non-zero orders, where

$$
\tilde{N}=N-\sum_{k=0}^{N}\left(1-\delta_{i}^{j}(t+k)\right),
$$

is the number of non-zero orders.

The vector reduction from $\mathbf{o}_{\mathbf{i}}^{\mathbf{j}}(\mathbf{t})$ to $\tilde{\mathbf{o}}_{\mathbf{i}}^{\mathbf{j}}(\mathbf{t})$ can be represented by the following change of variable:

$$
\mathbf{o}_{i}^{j}(t)=M \tilde{\mathbf{o}}_{\mathbf{i}}^{\mathbf{j}}(\mathbf{t}),
$$

where $M \in \mathbb{R}^{(N+1) \times(\tilde{N}+1)}$ is a matrix that reduces the dimension of the order vector $\mathbf{o}_{i}^{j}(t)$ depending on the value of $\delta_{i}^{j}(t)$. It is defined as

$$
M(i, j)= \begin{cases}1 & \text { if } \delta_{i}^{j}(t)=1 \wedge i=j \\ 0 & \text { if } \delta_{i}^{j}(t)=0 \vee i \neq j .\end{cases}
$$

As direct consequence, $\tilde{\mathbf{o}}_{\mathbf{i}}^{\mathbf{j}}(\mathbf{t})$ contains only non-null components, i.e., the orders that are non-zero.

Taking into account the integer nature of the variable $\delta_{i}^{j}(t)$, the resulting optimization problem is a mixed integer one (MIP). There are different techniques to solve them like branch and bound [29], genetic algorithms [30] or the cutting-plane method [31]. In this article, an exhaustive search approach has been implemented. This means that the optimization problem is solved for each possible realization of $\left\{\delta_{i}^{j}(t), \ldots, \delta_{i}^{j}(t+\right.$ $N)\}$. This way, Boolean variables are removed from the optimization. The optimal solution corresponds with the combination of the values of $\left\{\delta_{i}^{j}(t), \ldots, \delta_{i}^{j}(t+N)\right\}$ that provides the minimal value of the objective function.

Remark 1. It is necessary to pay special attention to the constraints while solving this problem. It is not possible to impose the whole matrix of constraints to the reduced vector $\tilde{\mathbf{o}}_{\mathbf{i}}^{\mathbf{j}}(\mathbf{t})$, so it is necessary to apply the change matrix $M$ to the matrix of constraints to impose them only to the considered control components. 


\section{2. $C C-M P C$}

The CC-MPC method is used to deal with the stock management problem because the constraints under consideration must be treated in stochastic manner. The optimization problem can be written as in (12), but subject to

$$
\begin{gathered}
\bar{G}_{i}(o, \bar{d}) \leq g_{i}, i=1,2, \ldots, m, \\
o \in D,
\end{gathered}
$$

where $D \subset \mathbb{R}^{n}, \bar{d}$ is a stochastic vector defined over a set $E \subset \mathbb{R}^{s}$, and $m$ is the number of constraints. It is assumed that there is a set of events $F$, formulated by subsets from $E$ and a probability distribution $P$, defined over $F$. Hence, for each $A \subset E$, the probability $P(A)$ is known. In addition, it is assumed that the functions $\bar{G}_{i}(o, \cdot): E \rightarrow \mathbb{R}, \forall o, i$ are random variables and the probability distribution $P$ is independent of the decision variable $o$.

For addressing this problem, it is necessary to rewrite the optimization problem as a deterministic equivalent in which the constraints are verified with a certain probability. These stochastic constraints are called chance constraints. These constraints can be formulated by two different manners as discussed below.

Joint Chance Constraint: The stochastic formulation of the joint chance constraints is given by the objective function (12), subject to

$$
\begin{gathered}
P\left(\bar{G}_{i}(o, \bar{d}) \leq g_{i}, \forall i\right)>1-\delta_{x}, \\
o \in D,
\end{gathered}
$$

where $\delta_{x} \in[0,1]$ is the risk that the constraints of the optimization problem are not fulfilled.

Individual Chance Constraint: Individual chance constraints are formulated with the same objective function (12), subject to

$$
\begin{gathered}
P\left(\bar{G}_{i}(o, \bar{d}) \leq g_{i}\right)>1-\delta_{x_{i}}, \forall i \\
o \in D,
\end{gathered}
$$

where it writes one equivalent by each stochastic constraint.

The aggregate demand $d(t)$ in $(14)$ includes a stochastic disturbance component given its uncertain nature. Due to the presence of these uncertainties, the constraints have a stochastic nature, i.e., they can not be written as deterministic ones. Therefore, the constraints can be expressed as

$$
P\left(s(t+k) \geq \mathrm{S}_{\min }\right) \geq 1-\delta_{\mathrm{s}}, \quad \forall k \in\{1, . ., N\},
$$

where $\delta_{\mathrm{s}}$ is the probability of having less stock than $S_{\min }$. This expression can be developed along $N$ and obtain the mean and standard deviation of the state.

For the first time instant along $N$ (i.e., $k=1$ ), it yields

$$
\begin{gathered}
P\left(s(t+0)+o(t+0)-d(t+0) \geq \mathrm{S}_{\min }\right) \geq 1-\delta_{\mathrm{s}}, \\
P\left(-d(t+0) \geq \mathrm{S}_{\min }-s(t+0)-o(t+0)\right) \geq 1-\delta_{\mathrm{s}}, \\
P(\underbrace{d(t+0)}_{\text {Random }} \leq \underbrace{-\mathrm{S}_{\min }+s(t+0)+o(t+0)}_{\text {Deterministic }}) \geq 1-\delta_{\mathrm{s}},
\end{gathered}
$$


which can be rewritten as

$$
\phi_{0}\left(-\mathrm{S}_{\min }+s(t+0)+o(t+0)\right) \geq 1-\delta_{\mathrm{s}},
$$

where $\phi_{0}(\cdot)$ is the cumulative distribution function of the random variable $d(t+0)$. The deterministic equivalent for this chance constraint is

$$
\begin{gathered}
-\mathrm{S}_{\min }+s(t+0)+o(t+0) \geq \phi_{0}^{-1}\left(1-\delta_{\mathrm{s}}\right), \\
-o(t+0) \leq-\mathrm{S}_{\min }+s(t+0)-\phi_{0}{ }^{-1}\left(1-\delta_{\mathrm{s}}\right) .
\end{gathered}
$$

For the next time instant along $N$ (i.e., $k=2$ ), it yields

$$
\begin{aligned}
& P\left(s(t+2) \geq \mathrm{S}_{\min }\right) \geq 1-\delta_{\mathrm{s}} \\
& P\left(s(t+1)+o(t+1)-d(t+1) \geq \mathrm{S}_{\min }\right) \geq 1-\delta_{\mathrm{s}} \\
& P\left((s(t+0)+o(t+0)-d(t+0))+o(t+1)-d(t+1) \geq \mathrm{S}_{\min }\right) \geq 1-\delta_{\mathrm{s}} \\
& \left.P(-d(t+0)-d(t+1)) \geq \mathrm{S}_{\min }-s(t+0)-o(t+0)-o(t+1)\right) \geq 1-\delta_{\mathrm{s}}, \\
& P\left(d(t+0)+d(t+1) \leq-\mathrm{S}_{\min }+s(t+0)+o(t+0)+o(t+1)\right) \geq 1-\delta_{\mathrm{s}} .
\end{aligned}
$$

Defining $\phi_{1}(\cdot)$ as the cumulative distribution function of the variable $d(t+0)+d(t+1)$ yields

$$
\begin{aligned}
& \phi_{1}\left(-\mathrm{S}_{\min }+s(t+0)+o(t+0)+o(t+1)\right) \geq 1-\delta_{\mathrm{s}}, \\
& -\mathrm{S}_{\min }+s(t+0)+o(t+0)+o(t+1) \geq \phi_{1}^{-1}\left(1-\delta_{\mathrm{s}}\right), \\
& o(t+0)+o(t+1) \geq \mathrm{S}_{\min }-s(t+0)+\phi_{1}^{-1}\left(1-\delta_{\mathrm{s}}\right), \\
& -o(t+0)-o(t+1) \leq-\mathrm{S}_{\min }+s(t+0)-\phi_{1}^{-1}\left(1-\delta_{\mathrm{s}}\right) .
\end{aligned}
$$

Iteratively (e.g., $k=3$ ) and according to the previous development, it can written as

$$
-o(t+0)-o(t+1)-o(t+2) \leq-\mathrm{S}_{\min }+s(t+0)-\phi_{2}{ }^{-1}\left(1-\delta_{\mathrm{s}}\right),
$$

where $\phi_{2}(\cdot)$ denotes the cumulative distribution function of the variable $d(t+0)+$ $d(t+1)+d(t+2)$. Generalizing for a prediction horizon $N$,

$$
-\left[\begin{array}{ccccc}
1 & 0 & 0 & \cdots & 0 \\
1 & 1 & 0 & \cdots & 0 \\
1 & 1 & 1 & \cdots & 0 \\
\vdots & & & & \vdots \\
1 & 1 & & \cdots & 1
\end{array}\right]\left[\begin{array}{c}
o(t+0) \\
o(t+1) \\
o(t+2) \\
\vdots \\
o(t+N-1)
\end{array}\right] \leq\left[\begin{array}{cc}
1 & 1 \\
1 & 1 \\
1 & 1 \\
\vdots & \vdots \\
1 & 1
\end{array}\right]\left[\begin{array}{c}
s(t+0) \\
-\mathrm{S}_{\min }
\end{array}\right]-\left[\begin{array}{c}
\phi_{0}{ }^{-1}\left(1-\delta_{\mathrm{s}}\right) \\
\phi_{1}{ }^{-1}\left(1-\delta_{\mathrm{s}}\right) \\
\phi_{2}{ }^{-1}\left(1-\delta_{\mathrm{s}}\right) \\
\vdots \\
\phi_{N-1}{ }^{-1}\left(1-\delta_{\mathrm{s}}\right)
\end{array}\right],
$$

where $\phi_{N-1}^{-1}\left(1-\delta_{\mathrm{s}}\right)$ is the cumulative distribution function of the random variable $d(t+0)+d(t+1)+d(t+2)+\cdots+d(t+N-1)$.

Remark 2. It is also possible to assume that the behavior of the disturbances can be adjusted as a function of a certain probability distribution. In [27], a normal distribution is used to characterized the behavior of the perturbations, with mean $\mu$ and standard deviation $\sigma$, i.e., $d(t)=N(\mu, \sigma)$. This assumption could be extended to other patterns or even work directly with historical data, like in this case. 


\section{Case Study and Results}

In this section, CC-MPC is going to be applied to manage the orders of two drugs available in the hospitals San Juan de Dios and Reina Sofía (both located at Córdoba, Spain). These drugs are not only expensive because of their prices but also because of their maintenance costs, since they must be stored in a fridge. Due to this fact, the reduction of their stock levels is a priority. Due to confidentiality reasons, the specific names and prices of these drugs are going to be omitted.

Regarding the controller, a prediction horizon $N=8$ days has been considered. The evolution of the stock is modeled by using the discrete-time linear model in (14). The orders of these drugs have a minimum amount of 4 units and the maximum has been set to 1000 . The prices of the drugs are respectively 227 and 298 euros per unit, respectively, and each order placed implies an additional cost of 2 euros. The deliveries of these drugs usually have a delay of 2 days with respect to the moment in which the order was placed. The initial values of the stock levels are 500 and 1520, respectively. For simplicity, neither storage cost nor storage limits have been considered at this stage of the proposed work. The only constraint implemented with respect to the stock is that the probability of stockout event has to be lower than 0.001 (i.e., it is requested a reliability level of $99.999 \%$ ). Finally, the demand term of (14) is non-deterministic. A probabilistic characterization of their behavior has been calculated for these drugs based on historical data.

If implemented in a hospital, the CC-MPC optimization problem should be solved daily to compute the optimal orders to steer the stock levels as desired. For this simulation, the stock reference (security stock) has been set to 2 . All optimization problems were computed by using linear programming routines ( $\mathrm{i}$ inprog in Matlab) on a machine with an Intel Core 2 Duo CPU with $3.33 \mathrm{GHz}$ and 8 GB RAM. The time needed to calculate the optimal sequence of actions per drug and day was below 10 seconds. If we take into account that the orders are recomputed once a day, it is possible to calculate optimal control actions for 8640 drugs a day with the current configuration, many more than those used in the hospitals.

The 360-days simulation scenario considered here is shown in Figures 2 and 3 for each drug. In black, the evolution of the stocks using CC-MPC is shown. In red, the real evolution of the stock according to the hospital data is shown. Tables 4 and 4 show a comparison of the behavior of these two drugs applying CC-MPC with the results registered by the hospitals in this period, considering the average level, standard deviation, and the number of orders. In this period the hospitals placed 9 orders for the drug 1 and 25 for the second one while the CC-MPC policy resulted in 5 and 15 orders respectively. The better timing of the CC-MPC orders and the optimally calculated order size are the key to explain the lower performance of the hospital. On average, the CC-MPC strategy would have resulted in 39 units of drug 1 and 770 of drug 2, i.e., 4 stocked units less of drug 1 and 91 of drug 2 with respect to the results registered by the hospital. Notice also that the CC-MPC strategy results in a slightly greater standard deviation of the stock levels, i.e., the fluctuations in the number of stocked items are a bit bigger with this policy. This is natural since less orders are placed with the CC- 

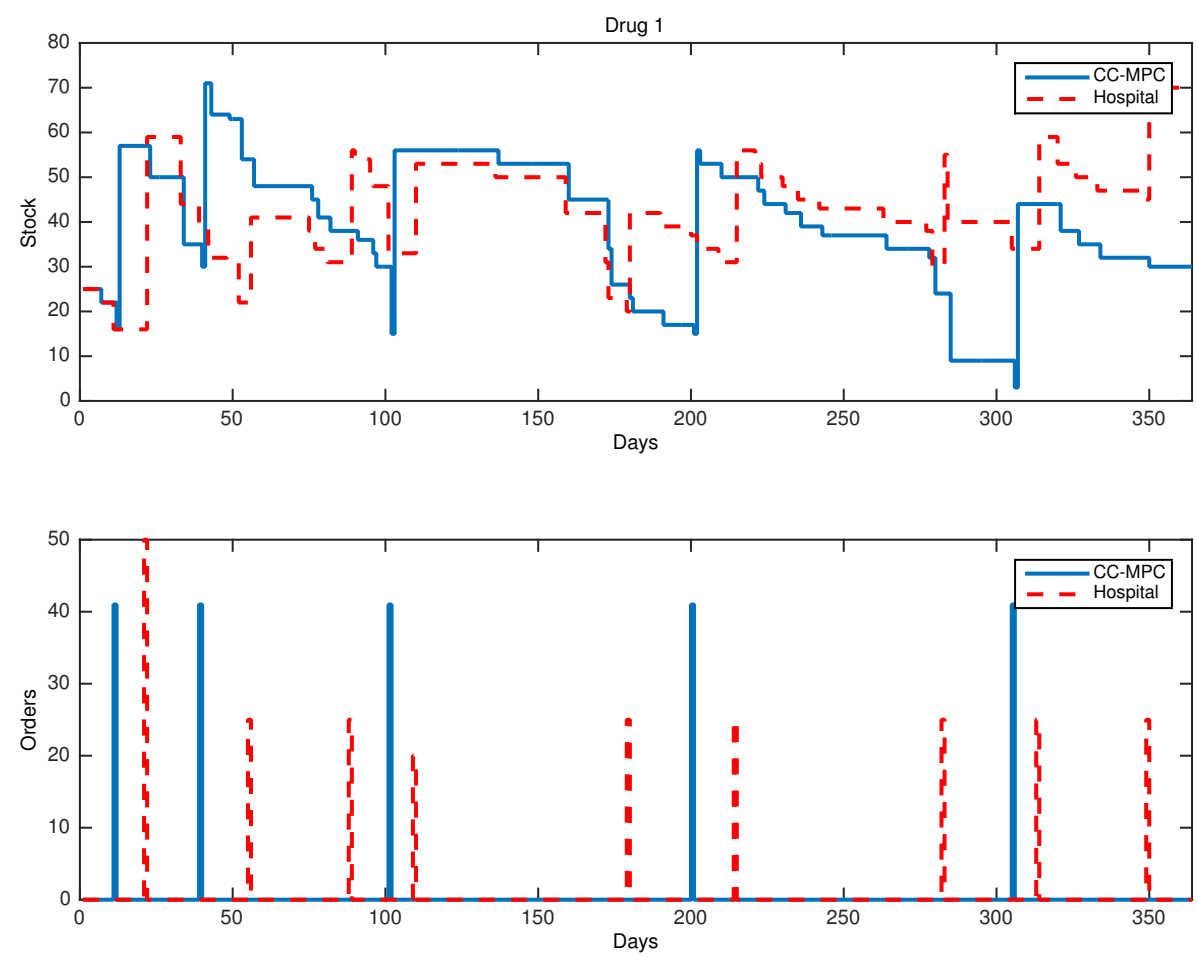

Figure 2: Real and simulated stock evolution and placed orders for drug 1.

MPC strategy. Finally, notice that no stockouts happened during the simulation period.

These results clearly show that the CC-MPC policy provides better results than the policy that is currently implemented in the hospital pharmacies. For the first drug, more than 1000 euros on average could be used for purposes other than having stock at the pharmacy with the same clinical results. In the case of drug 2 this amount is 27118 euros. Another noteworthy point is that the staff at the pharmacy department is freed partially from the duties related to the placement and reception of orders. In both cases the CC-MPC placed $40 \%$ less orders than the policy followed by the hospital. Finally, note that a more aggressive tuning of the controller could be used to reduce these values at the cost of higher stockout risks.

Table 1: Comparison of the behavior of the drug 1 applying CC-MPC and hospital policy.

\begin{tabular}{|l|l|l|l|l|}
\hline Approach & Orders & Stock-out & Mean & Desviation \\
\hline CC-MPC & 5 & 0 & 39 & 14 \\
\hline Hospital historical data & 9 & 0 & 43 & 11 \\
\hline
\end{tabular}



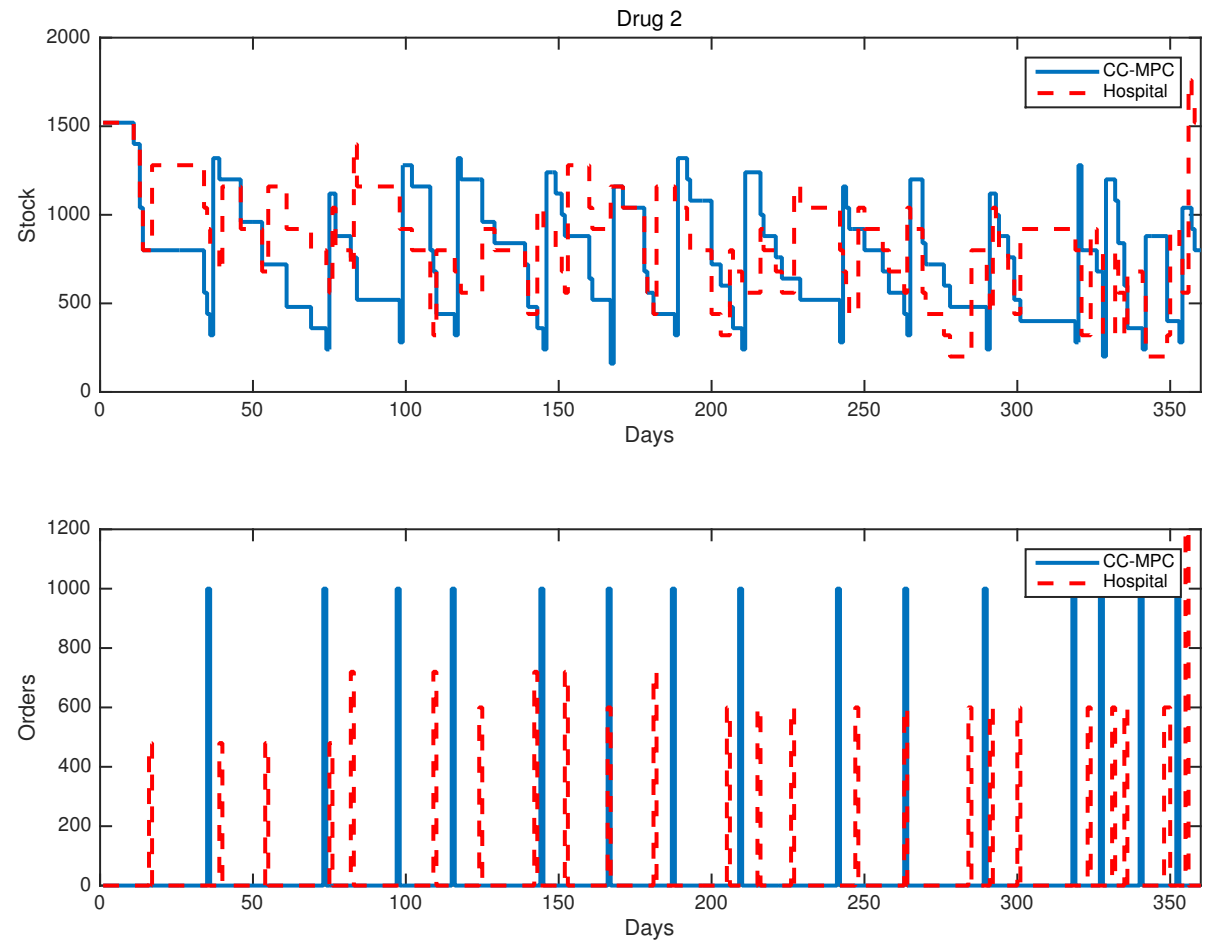

Figure 3: Real and simulated stock evolution and placed orders for drug 2.

Table 2: Comparison of the behavior of the drug 2 applying CC-MPC and hospital policy.

\begin{tabular}{|l|l|l|l|l|}
\hline Approach & Orders & Stock-out & Mean & Desviation \\
\hline CC-MPC & 15 & 0 & 770 & 316 \\
\hline Hospital historical data & 25 & 0 & 861 & 313 \\
\hline
\end{tabular}

\section{Conclusions}

Stock management is one of the main tasks of the pharmacy department of a hospital. It is a complex problem due to the uncertainty in the drug demand and the variety of constraints to be considered. In this work, a control technique to deal with this problem has been described and assessed using real data from two Spanish hospitals. The proposed strategy is based on MPC, which allows the fulfillment of the management objectives while taking into account the different operational constraints. The use of chance constraints in combination with MPC makes possible to guarantee the availability of drugs for the patients with a certain level of risk that is explicitly set. 
The results of the simulations show that CC-MPC allows reducing the average drug stock level and the work burden in the pharmacy while satisfying the drug demand. The key for the improvement lies on the better time and order size of the proposed approach. Therefore, this control strategy leads to a more efficient use of the resources by the pharmacy department.

Currently, this control approach is translated to C language as a software tool to be implemented in the hospitals that collaborate in this work.

\section{References}

[1] T. Bermejo, B. Cuña, V. Napal, E. Valverde, The hospitalary pharmacy specialist handbook (in Spanish), Spanish Society of Hospitalary Pharmacy, 1999.

[2] S. Tayur, R. Ganeshan, M. Magazine, Quantitative models for supply chain management, Kluwer Academic Publisher, 1999.

[3] A. M. Brewer, K. J. Button, D. A. Hensher, Handbook of logistics and supplychain management, Pergamon, 2001.

[4] S. Çetinkaya, C. Lee, Stock replenishment and shipment scheduling for vendormanaged inventory systems, Management Science 46 (2) (2000) 217-232.

[5] J. Dong, D. Zhang, A. Nagurney, A supply chain network equilibrium model with random demands, European Journal of Operational Research 156 (1) (2004) 194-212.

[6] G. Cachon, Stock wars: Inventory competition in a two-echelon supply chain with multiple retailers, Operations Research 49 (5) (2001) 658-674.

[7] S. Rezapour, R. Farahani, Strategic design of competing centralized supply chain networks for markets with deterministic demands, Advances in Engineering Software 41 (5) (2010) 810-822.

[8] E. F. Camacho, C. Bordons, Model Predictive Control in the Process Industry. Second Edition, Springer-Verlag, London, England, 2004.

[9] W. Wang, D. E. Rivera, K. G. Kempft, K. D. Smith, A model predictive control strategy for supply chain management in semiconductor manufacturing under uncertainty, in: Proceedings of the 2004 American Control Conference, Vol. 5, Boston (MA), 2004, pp. 4577-4582.

[10] W. Wang, D. E. Rivera, K. G. Kempf, A novel model predictive control algorithm for supply chain management in semiconductor manufacturing, in: Proceedings of the 2005 American Control Conference, Vol. 1, Portland, Oregon, 2005, pp. 208-213.

[11] J. M. Maestre, D. Muñoz de la Peña, E. F. Camacho, Distributed model predictive control based on a cooperative game, Optimal Control Applications and Methods 32 (2) (2011) 153-176. 
[12] J. M. Maestre, D. Muñoz de la Peña, E. F. Camacho, T. Alamo, Distributed model predictive control based on agent negotiation, Journal of Process Control 21 (5) (2011) 685-697.

[13] C. Stoica, M. Arahal, D. Rivera, D. Rodríguez-Ayerbe, P.and Dumur, Application of robustified model predictive control to a production-inventory system, in: Proceedings of the 48th Conferece on Decision and Control (CDC), Shangai, 2009, pp. 3993-3998.

[14] H. Rasku, J. Rantala, H. Koivisto, Model reference control in inventory and supply chain management - The implementation of a more suitable cost function, Springer Netherlands, 2006, pp. 111-116.

[15] D. Muñoz de la Peña, A. Bemporad, T. Alamo, Stochastic programming applied to model predictive control, in: Proceedings of the 44th IEEE Conference on Decision and Control (CDC) and European Control Conference (CDC-ECC), Seville, Spain, 2005, pp. 1361-1366.

[16] A. Prekopa, On probabilistic constrained programming, in: In Proceedings of the Princeton Symposium on Mathematical Programming, Princeton University Press, NJ, 1970, pp. 113-138.

[17] P. Kall, J. Mayer, Stochastic linear programming, Springer, New York, NY, 2005.

[18] A. Geletu, M. Klöppel, H. Zhang, P. Li, Advances and applications of chanceconstrained approaches to systems optimisation under uncertainty, International Journal of Systems Science 44 (7) (2013) 1209-1232.

[19] A. Schwarm, M. Nikolaou, Chance-constrained model predictive control, AIChE Journal 45 (8) (1999) 1743-1752.

[20] J. Grosso, C. Ocampo-Martinez, V. Puig, B. Joseph, Chance-constrained model predictive control for drinking water networks, Journal of Process Control 24 (5) (2014) 504-516.

[21] W. Langson, I. Chryssochoos, S. V. Raković, D. Q. Mayne, Robust model predictive control using tubes, Automatica 40 (1) (2004) 125-133.

[22] P. O. M. Scokaert, D. Q. Mayne, Min-max feedback model predictive control for constrained linear systems, IEEE Transactions on Automatic Control 43 (8) (1998) 1136-1142.

[23] G. Schildbach, L. Fagiano, C. Freic, M. Morari, The scenario approach for stochastic model predictive control with bounds on closed-loop constraint violations, Automatica 50 (12) (2014) 3009-3018.

[24] J. Maestre, L. Raso, P. van Overloop, B. De Schutter, Distributed tree-based model predictive control on a drainage water system, Journal of Hydroinformatics 15 (2) (2013) 335-347. 
[25] P.-J. van Overloop, S. Weijs, S. Dijkstra, Multiple model predictive control on a drainage canal system, Control Engineering Practice 16 (5) (2008) 531-540.

[26] J. M. Grosso, J. M. Maestre, C. Ocampo-Martinez, V. Puig, On the assessment of tree-based and chance-constrained predictive control approaches applied to drinking water networks, in: Proceedings of the 19th IFAC World Congress, IFAC, Cape Town, South Africa, 2014, pp. 6240-6245.

[27] J. Maestre Torreblanca, P. Velarde, I. Jurado, C. Ocampo-Martinez, I. Fernandez, B. Isla Tejera, J. del Prado Llergo, An application of chance-constrained model predictive control to inventory management in hospitalary pharmacy, in: 53rd IEEE Conference on Decision and Control, CDC 2014, Los Angeles, CA, USA, December 15-17, 2014, pp. 5901-5906.

[28] P. Velarde, J. Maestre Torreblanca, I. Jurado, I. Fernandez, B. Isla Tejera, J. del Prado Llergo, Application of robust model predictive control to inventory management in hospitalary pharmacy, in: Proceedings of the 2014 IEEE Emerging Technology and Factory Automation, ETFA 2014, Barcelona, Spain, September 16-19, 2014, pp. 1-6.

[29] E. Lawler, D. Wood, Branch-and-bound methods: A survey, Operations Research 14 (4) (1966) 699-719.

[30] V. Summanwara, V. Jayaramana, B. Kulkarnia, H. Kusumakarb, K. Guptab, J. Rajesh, Solution of constrained optimization problems by multi-objective genetic algorithm, Computers \& Chemical Engineering 26 (10) (2002) 1481-1492.

[31] W. Cook, R. Kannan, A. Schrijver, Chvátal closures for mixed integer programming problems, Mathematical Programming 47 (1) (1990) 155-174. 